\title{
Pulsed Blue Laser Diode Thermal Desorption Microplasma Imaging Mass Spectrometry
}

\author{
Alexander Knodel ${ }^{\dagger}$, Ulrich Marggraf ${ }^{\dagger}$, Edeltraut Hoffmann-Posorske ${ }^{\dagger}$, Sebastian Burhenn ${ }^{\#}$, Sebastian \\ Brandt $^{\dagger}$, Norman Ahlmann ${ }^{\dagger}$, Daniel Foest ${ }^{\dagger}$, Kristina Lorenz ${ }^{\dagger, \S}$, Joachim Franzke ${ }^{\dagger}{ }^{*}$ \\ 'Leibniz-Institut für Analytische Wissenschaften - ISAS - e.V., Bunsen-Kirchhoff-Straße 11, 44139 Dortmund, Germany \\ "Experimental Physics II, Faculty of Physics and Astronomy, Ruhr-University Bochum, 44801 Bochum, Germany \\ §Institute of Pharmacology and Toxicology, University of Würzburg, 97078 Würzburg, Germany \\ *E-mail: franzke@isas.de. Phone: +49 (0)231 1392-174. Fax: +49 (0)231 1392-120.
}

\begin{abstract}
An ambient air laser desorption, plasma ionization imaging method is developed and presented using a pulsed laser diode for desorption and the flexible microtube plasma for ionization of the neutral desorbate. Inherent parameters such as the laser pulse frequency and pulse width are optimized to the imaging application. For the desorption substrate, copper spots on a copper-glass sandwich structure are used. This novel design enables imaging without ablating the metal into the mass spectrometer. On this substrate, fixed calibration markers are used to decrease the positioning error in the imaging process, featuring a 3D offset correction within the experiment. The image is both screened spot-by-spot and per line scanning at a constant speed, which allows direct comparison. In spot-by-spot scanning, a novel algorithm is presented to unfold and to reconstruct the imaging data. This approach significantly decreases the time required for the imaging process, which allows imaging even at decreased sampling rates and thus higher mass resolution. After the experiment, the raw data is automatically converted and interpreted by a second algorithm, which allows direct visualization of the image from the data, even on low-intensity signals. Mouse liver microtome cuts have been screened for dehydrated cholesterol, proving good agreement of the unfolded data with the morphology of the tissue. The method optically resolves $30 \mu \mathrm{m}$, with $30 \mu \mathrm{m}$ diameter copper spots and a $10 \mu \mathrm{m}$ gap. No conventional chemical matrices or vacuum conditions are required.
\end{abstract}

In mass spectrometry imaging, secondary ion mass spectrometry $(\text { SIMS })^{1}$ as well as matrix-assisted laser desorption ionization (MALDI) ${ }^{2}$ have been excessively used to screen biological samples. In MALDI, a pulsed UV or IR laser is used on a matrix-coated substrate. The key concept of MALDI is its matrix, which absorbs the laser light and ejects both neutral and ionized molecules from the tissue. The matrix is commonly evaporated onto the substrate and the tissue under vacuum conditions. Likewise, the experiment is also carried out at vacuum conditions. These conditions are both boon and bane, as they allow high resolution imaging, but also complicate the experiments.

On the other hand, numerous, often hyphenated ambient mass spectrometry methods have emerged in the past decade. These methods range from atmospheric pressure MALDI (APMALDI), commonly used with commercialized imaging probes $^{3,4,5}$ to laser ablation methods coupled to flowing atmospheric pressure afterglow (FAPA) ${ }^{6}$ or direct analysis in real time (DART) ${ }^{7,8}$ ionization. FAPA is often combined with thinlayer chromatography (TLC) or an ablation cell. ${ }^{9}$ These ablation cells may significantly impact the working pressure, if they are not supplied with a gas flow matching to the sucking properties of the mass spectrometer. Commercialized TLC plates have also been used in combination with low-temperature plasma $(\mathrm{LTP})^{10}$ ionization or plasma-assisted (multiwavelength) laser desorption ionization (PALDI). ${ }^{11,12}$ Recently, plant metabolites were desorbed from native tissue and imaged using a continuous-wave (cw) UV laser and an LTP, even combined with a conventional matrix. ${ }^{13,14}$

Tandem mass spectrometry was carried out by laser diode thermal desorption, atmospheric pressure chemical ionization (LDTD-APCI-MSMS) ${ }^{15-17}$ on metallized well plates using human plasma, fish brain tissue, and metformin in different biological samples. The sample preparation includes the extraction of the target analytes from the biological plasma. However, imaging on real samples, despite the TLC plates, was not often part of the publications. Despite, major differences to this method are the laser parameters, type of ionization source as well as the choice of desorption substrate that will be discussed at the end of the manuscript. Furthermore, silver ${ }^{18}$ and gold ${ }^{19}$ were used in a surface-assisted laser desorption ionization (SALDI) approach, also combined with electrospray ionization. $^{20}$

Previously, standardized copper-glass substrates were introduced to a diode laser, plasma ionization interface ${ }^{21}$ that enabled precisely controlled desorption of cholesterol and other standards from a cavity structure. These copper-glass slides were also used in the context of several hydrophobic analytes and different lipid classes. ${ }^{22}$ Among others, two crucial physical properties were highlighted in these publications: First, the molten copper was trapped in the glass as consequence of the laser heating, resulting in desorption of neutral desorbate. Second, several analytes have shown different desorption thresholds in terms of different laser fluences, making the process of desorption temperature dependent. In general, these results have verified that laser desorption plasma ionization works under ambient air conditions, even with laser diodes at rather low intensities compared to pulsed Nd:YAG-lasers.

However, the cavity structure with micrometer height did not allow real sample to be screened, as the tissue could not be placed fully plane on the substrate. As a consequence, the cavity structure was developed into a grid of planar copper spots, again formed on the microscope slide. In addition, mandatory calibration markers were added to the design that allow to online level the substrate in the experiment, which consequently reduces the positioning error in the imaging application. 
It is worth noting that copper spots are prepared on a glass substrate at first, which will be used to screen liver samples in a second step. That way, the metal will absorb the laser light and will assist the heat propagation to the tissue. This in contrast to conventional matrix- and surface-assisted laser desorption ionization methods, in which the dopant is included in the ionization process. For instance, MALDI ions are most likely formed with the analyte coupled to the matrix. In the metal-assisted approaches previously mentioned, even if no matrix was used, metal clusters as well as adducts were observed in the mass spectrum. In this sense, the method presented in this work is considered matrix-free but assisted by copper spots, which only increase the local surface temperature by more efficient laser absorption but do not take part in the ionization process.

Even though proteins ${ }^{23}$ and sphingolipids ${ }^{24,25}$ have been screened, not many sterols ${ }^{26}$ are imaged in mass spectrometry despite their significance, yet. This circumstance may relate to the issue that electrospray ionization does not seem to well ionize dehydrated cholesterol without further sample manipulation, in contrast to plasmas. However, disturbances in the cholesterol levels are associated to numerous diseases, such as nonalcoholic fatty liver or cardiovascular diseases and neurodegeneration. ${ }^{27,28,29}$ The importance of a target analysis of cholesterol was also recently highlighted by Cologna ${ }^{30}$. The author presented cholesterol analysis in SIMS, MALDI and DESI. All in all, these aspects outline the necessity of novel methods for imaging of biological samples.

\section{EXPERIMENTAL METHODS}

\section{Diode Laser Desorption, Plasma Ionization MSI (DLD-PI MSI)}

The experimental assembly is shown in Fig. 1, which shows the coupling of a pulsed laser diode to a miniaturized, flexible microplasma for the imaging of biological tissue.

For laser desorption, a grid of copper spots on glass substrates was used for the desorption of non-ionized molecules, the desorbate. The diode laser was applied to the glass-side of the substrate, which transmits the laser beam through the glass to the copper spots, sometimes referred to as transmission geometry. The copper spots absorb the light and heat the tissue. The heated tissue consequently ejects neutral desorbate from the surface. For laser application, each spot is centered $2 \mathrm{~mm}$ in front of the mass spectrometers inlet. The laser is refocused to each spot by an offset correction within the experiment.

The starting point as well as the coordinates of the calibration markers need to be set manually with the software. The stages absolute position is first stored into the internal memory and subsequently read from the buffer by the software. From the chosen starting point, the stage automatically travels to the next spot in each row and column, limited by the user-given region of

interest

(ROI).

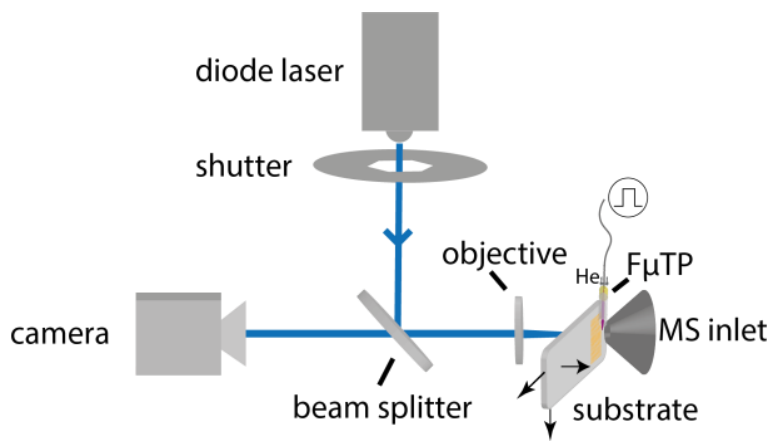

Fig. 1. Experimental arrangement for ambient air, diode laser desorption imaging with consecutive $\mathrm{F} \mu \mathrm{TP}$ ionization mass spectrometry. The substrates used are copper spots on conventional microscope glass slides. The laser was applied to the glass-side of the substrate, on-axis to the mass spectrometers orifice. The F $\mu$ TP was placed in between the mass spectrometers orifice and the desorption substrate.

The script performs a correction for several linear errors emerging from tilts of the spots and the experiment itself using calibration markers on the substrate. As the laser is applied from the glass slide, topological inhomogeneity of the tissue does not contribute to the laser focus error. For an ROI of $5 \mathrm{~mm} \times 5 \mathrm{~mm}$, the laser focus diverges approximately $(32 \pm 6) \mu \mathrm{m}$, being corrected using the aforementioned calibration markers. Notably, the effectiveness of the laser focus correction depends on the position of the calibration markers chosen on the desorption substrate. For instance, calibration marker close to the starting point allow more precise error correction than markers far from the starting point. The stage moves unidirectional to avoid hysteresis effects from stage moving, that would result in higher error. These efforts decrease the additive positioning error down to less than $0.5 \%$ within the axis of movement. The error on the axis perpendicular to the moving direction, thus within the offset-corrected plane, is estimated to be below $10 \mu \mathrm{m}$ for a full range scan $(<0.04 \%)$. It should be highlighted that the calibration procedure is executed with deactivated transients of the high voltage driver, which improve the precision of the stage.

The functionality of the software further covers custom laser application times, both spot and line scanning with custom scan speeds or step sizes with automated tilt correction, user-defined ROIs, control of cw-operated laser diode power and "triggering" the mass spectrometer - the trigger is considered as a start pulse for the measurement. The mapped 2D coordinates are exported into a text file.

In both spot-by-spot and line scanning, each line of spots is stored into a single file. For that reason, a post processing algorithm is required, that unfolds the raw data into single spot information. This effort also reduces the experimental time.

The full screening of the copper substrates presented in Diode Laser Desorption, Plasma Ionization MSI (DLD-PI MSI) takes between five and eight hours in total. The post processing is carried out in less than an hour, including preparing the final images. A full description of the sofware is given in the Supplementary as well as in Supplementary Fig. S1 and S2. The method resolves $30 \mu \mathrm{m}$ in spot-by-spot scanning. 


\section{Hardware}

A data acquisition (DAQ) interface was used for analog and digital input/output signaling (USB-6341 BNC DAQ, National Instruments, USA).

Each experiment is initialized by a pull-down pulse to the mass spectrometer through the start-in socket. A $5 \mathrm{~V}, 16 \mathrm{~mA}$ output of the DAQ interface is fed to an opto-isolator (SFH6012) coupled to a $390 \Omega$ resistance, which provides a pull-down logic of the 5V TTL-signal of the mass spectrometer (start-in socket).

For remote control, the BNC modulation input of the laser controller needs to be used. The diode is pulsed using a waveform generator (HM8150, Rohde \& Schwarz Value Instruments, formerly Hameg, Germany). The controller translates the pulse amplitude with $400 \mathrm{~mA} / \mathrm{V}$ and is set above laser threshold. The laser pulse frequency, width and a voltage offset can be set from the waveform generator, as the diode controller replicates the input signal within the controllers limit. The shutter needs to be set to external triggering using TTL logic for external operation. The electronical stage (T-LS28-M, Zaber Technologies, Canada) is remotely controlled as well, using the RS-232 protocol and readily available Zaber VIs for LabVIEW (version 1.4.0). ${ }^{33}$

\section{Ionization Source}

The ionization source is described in detail in the Supplementary.

\section{Diode Laser}

For laser desorption of molecules from biological tissue, a $450 \mathrm{~nm}$ central wavelengths diode laser is used (diode: LDM448-3500-C, Lasertack, Germany ${ }^{34}$; controller: ITC540, Profile, Germany). The diode outputs $3.5 \mathrm{~W}$ at maximum. Two anamorphic prisms were used to correct beam divergence of the diode. The laser beam was guided through a beam splitter, which splits the beam to the glass-side of the substrate and the substrates reflection to the camera (UK-1158, ABS-Jena, Germany). An electrical shutter (shutter: Sh 05, Thorlabs, USA; controller: SC10, Thorlabs, USA) was used to block the beam of the laser. The laser beam was focused using a lens system (objective) in a lens revolver (LPL 40x / 0.65, all parts dismounted from OKM173, Kern, Germany). For alignment, a 5x objective was used (Kern Plan5x/0.11 from OKM173, Kern, Germany). The calibration of the diode laser current to the laser power was performed and described in a previous publication ${ }^{22}$. Although the described optics changed, the cw laser output power does not change significantly. The laser spot was recorded by a CCD camera (1/3" S/W A1-Pro, Conrad Electronics, Germany), shown in Supplementary Fig. S3. An average laser intensity is hard to provide, as the pulse is not rectangular. Based on cw-power meter measurements and the pulse profile, the maximum laser intensity is assumingly below $200 \mathrm{~W} / \mathrm{mm}^{2}$. A house-made photodiode amplifier connected to an oscilloscope (DPO2012, Tektronix, USA) is used to check pulse signals of the laser diode, as shown in Supplementary Fig. S4-S6. The effects are discussed in the results section. The laser light is attenuated by $99.7 \%$ using neutral density filters in a wheel attenuator (10CWA168, Standa, Lithuania). The laser diode is operated with a pulse frequency of $20 \mathrm{kHz}$ and a pulse width of $20 \mu \mathrm{s} \quad$ (duty cycle $40 \% \quad$ : $\quad 60 \%$ ).

\section{Safety Considerations}

The diode laser used in these experiments is a class 4 laser. Safety precautions need to be taken by wearing protective goggles, in accordance to the laser power and wavelength of the used laser. As the plasma operates at high voltage, a safety interlock was installed in the power supply to ensure user safety. Please consider safety data sheets for safe operation and handling of all used devices.

\section{Mass Spectrometry System}

The ions were detected by a triple quadrupole mass spectrometer (TSQ Vantage, Thermo Fisher Scientific, USA). For data acquisition, Xcalibur software version 2.0 (Thermo Fisher Scientific, USA) was used. Full-scan acquisition and selected ion monitoring (SIM) were carried out in positive ion mode. Both scans were performed on the third quadrupole (Q3). Sampling rates of $60 \mathrm{~Hz}$ were achieved in SIM and neither internal averaging nor micro scans were used. The Q3 was set at a resolution of 0.6 full width at half maximum (FWHM) with a mass window of $\delta \mathrm{m} / z=1.5$ and a cycle time of $10 \mathrm{~ms}$. The capillary temperature maintained at $250{ }^{\circ} \mathrm{C}$. The mass and resolution calibration were performed with the commercial available calibration solution (Pierce Triple Quadrupole Calibration Solution, 88325, Thermo Fisher Scientific, USA) by the use of a commercial IonMax API source (equipped with HESI probe). A Paul ion trap mass spectrometer (LCQ Deca XP, Thermo Fisher, USA) in full-scan acquisition and positive ion mode from $\mathrm{m} / \mathrm{z}, 100$ to $\mathrm{m} / \mathrm{z}, 1000$ was used to measure the spectra on the liver, brain and kidney tissue. The Automatic Gain Control (AGC) was used with a target ion current of $5 \cdot 10^{7}$ and $20 \mathrm{~ms}$ cut-off injection time without micro scans. The actual injection times were in the range of $0.5-5 \mathrm{~ms}$. The tube lens offset voltage was set to $-16 \mathrm{~V}$, the capillary voltage to $16 \mathrm{~V}$ and the capillary temperature maintained at $250{ }^{\circ} \mathrm{C}$. The entrance lens voltage in the ion optics was set to $-60 \mathrm{~V}$. The electron multiplier detector was operated at $-850 \mathrm{~V}$. A nano electrospray ionization source with calibration mix solutions (Caffeine, Met-Arg-Phe-Ala [MRFA] and Ultramark 1621) was used for mass calibration of this device.

\section{Sample Preparation}

Mouse liver, kidney and brain (FVB mouse, female, Janvier Labs, France) were flash-frozen in liquid nitrogen and stored at $-80{ }^{\circ} \mathrm{C}$ until usage. The mice were maintained in accordance with federal guidelines and handled corresponding to the Committee on Animal Research of the regional government (Landesamt für Natur, Umwelt und Verbraucherschutz Nordrhein-Westfalen LANUV-NRW). No mice were killed for this purpose but the organs were provided supplementary. Standard chow diet and water were offered ad libitum; for housing sterilized plastic cages under specific pathogen-free conditions were used; as housing conditions $(22 \pm 2){ }^{\circ} \mathrm{C}, 12 / 12$ light/dark cycle, $(55 \pm 10) \%$ humidity were maintained. Before cutting the tissue, the samples were stored in a $-20^{\circ} \mathrm{C}$ freezer for at least two hours to avoid fissures. A cryomicrotome cutter (CryoStar NX70, Thermo Fisher Scientific, USA) was used to cut the tissue. The samples were mounted on a sample holder using frozen section medium (NEG 50, Thermo Scientific Richard-Allan Scientific, USA), frozen to $-60^{\circ} \mathrm{C}$ for 10 minutes. Final microtome cuts were made at a thickness of $(10.0 \pm 0.5) \mu \mathrm{m}$. Each time a cut was taken out of the freezer, a vacuum desiccator 
(Concentrator Plus, Eppendorf, Germany) was used to minimize water residuals on the tissue. No further sample preparation is required.

Hematoxylin and eosin $(\mathrm{H} \& \mathrm{E})$ stains were formed from frozen $\left(-20^{\circ} \mathrm{C}\right)$ microtome cuts on glass substrates (SuperFrost Plus, Thermo Scientific, USA) using a tissue stainer (ST5020 Multistainer, Leica Biosystems, Germany) based on ROTI Histol (Roth, Germany), eosin Y, Mayers hematoxylin solution (both Sigma-Aldrich, USA), acetic acid, ethanol (100\%, p.a., both Roth, Germany) and (distilled) water (18.2 M $\Omega \mathrm{cm}$, Elga Purelab Flex, Veolia Water Technologies, Germany). The recipe for the production of the stains is listed in Supplementary Table S7. After staining, cytosol (Cytoseal XYL, Thermo Scientific, USA) and cover glass (20 mm x $20 \mathrm{~mm}$, Thermo Scientific, USA) were used. The cytosol was dried over night. The stained tissue was observed (DM6 B, Leica, Germany) with a white balance and a shader correction.

\section{Preparation of Copper Spots on Glass}

The custom-made substrate consists of a grid of circular copper spots with a fixed gap. The production process can be split into the substrate preparation, the sputtering process and lithography, explained in detail in the Supplementary.

The final design includes circular copper spots on conventional microscope glass slides and calibration markers for softwarecontrolled levelling. The spots are $(30 \pm 1) \mu \mathrm{m}$ in diameter with gaps of $(10 \pm 0.4) \mu \mathrm{m}$ between each spot. An image of the spots and a calibration marker are shown in Supplementary Fig. S8. An macroscopic image of the substrate is shown in the TOC graphic as well.

\section{RESULTS AND DISCUSSION}

Fundamental research was performed in previous publications using cavity-like copper-glass sandwich structures and standards. $^{21,22}$ The base point of the design was to confine the droplet spread on the substrate and to standardize the laser desorption properties. Standards tested ranged from cholesterol, fatty acids, sterols, phospholipids and triacylglycerol to different hydrophobic compounds such as the non-steroidal anti-inflammatory drugs ibu- and ketoprofen. These results have proven the applicability of ambient air cw diode laser desorption plasma ionization, especially promising for the detection of different lipid classes.

However, in terms of biological tissue, the cavity-like design is not optimal. In this sense, a flat structure is required to keep the structural integrity of the tissue. Further, the thermal heat propagation across the substrate that triggers desorption requires a controllable frame to draw conclusions on mass spectrometric data. Furthermore, calibration markers are used to level the substrate and align it to the laser diode.

One way to reduce the heat propagation is to use small copper spots matched to the laser spot size, as the surrounding glass has a lower heat conduction than copper. This design also provides the opportunity to identify the location of laser application more easily, especially when dealing with small laser spots.

However, preliminary experiments with animal tissue have shown that this concept is not sufficient to confine the heat propagation, especially in the realm of imaging applications. The reason is that heat propagates through the lowest thermal resistance, which is from the copper spots through the microtome cut, but not the glass. One way to reduce these effects is to shorten the laser application time, such as having pulses instead of a cw laser. In this way, passive cooling of the substrate is enabled after each laser pulse application. Laser diodes can be operated in $\mathrm{cw}$ to kilohertz-pulsed regimes. However, one drawback from pulsing laser diodes is the loss of laser power, as a higher modulation frequency and pulse width always comes with a lower laser power output. In fact, this issue also applies to diode-pulsed ND:YAG lasers. This is systematically shown in Supplementary Fig. S4 and S5. The range of modulation is commonly addressed as the $3 \mathrm{~dB}$ bandwidth, which translated into $70,7 \%$ of the maximum modulation amplitude. For better understanding, Fig. S6 shows a modulation signal with a high amplitude. The maximum amplitude is commonly measured in low-frequency or $\mathrm{cw}$ operation. For the diode in this experiment, the $3 \mathrm{~dB}$ bandwidth is thus approximately $30 \mathrm{kHz}$. This effect is also not related to the waveform function generator, which offer rise times of $8 \mathrm{~ns}$, but to the laser diode itself. Even though there are pulsed diodes commercially available up to the megahertz regime ${ }^{36}$, these diodes only offer low laser power output up to milliwatts but fixed pulsed width of $10 \mathrm{~ns}$, for instance. At $1 \mathrm{~W}$ of laser output power, pulse frequencies of $50 \mathrm{kHz}$ are a common limitation. Another setscrew is the pulse width, sometimes addressed as the duty cycle of the laser pulses. The limits of these two properties are given by the rise time of the laser diodes signal on the lower limit and two pulses coinciding on the upper limit. Thus, the $3 \mathrm{~dB}$ bandwidths are $10 \mu \mathrm{s}$ and $40 \mu \mathrm{s}$, having a laser pulse frequency of $20 \mathrm{kHz}$. On the one hand, typical rise times of the laser diode used are around $(15 \pm 3) \mu \mathrm{s}$. On the other hand, laser diode controller can output rise times of $(9.5 \pm 0.5) \mu \mathrm{s}$ pulses. These limits dictate the diameter of desorption, which is addressed in Supplementary Fig. S9.

With a frequency close to cw-operation, the laser spot diameter is almost $100 \mu \mathrm{m}$, taken with a duty cycle of $50 \%$ as shown in Supplementary Fig. S9a. This diameter decreases down to $50 \mu \mathrm{m}$ with frequencies up to $20 \mathrm{kHz}$. Even though this diameter is considered an upper limit with that frequency, the pulse width can be adapted as well. At a frequency of $20 \mathrm{kHz}$, the pulse width is varied, as shown in Supplementary S9b. The red point highlights the same experimental conditions in both subfigures. The diameter decreases down to $30 \mu \mathrm{m}$ with pulse widths of $5 \mu$ s. However, a pulse width below $20 \mu$ s comes with a significant decrease in the measured modulation amplitude, as previously mentioned. This effect emerges from cutting both the rising and falling edge of the signal. Practically, these pulses would show a background cw-operation mode with pulse spikes on top of it. In other words, the modulation amplitude would be decreased artificially. Thus, the surface would be less heated and less molecules were desorbed. Therefore, a pulse frequency of $20 \mathrm{kHz}$ with a pulse width of $20 \mu \mathrm{s}$ is chosen. With these efforts, the heat propagation on the matrix was successfully confined. Considering the laser pulse frequency and pulse width, an effective laser application time of $0.4 \mathrm{~s}$ each copper spot is achieved.

On basis of these parameters, the laser is tested different grid designs. In this sense, different spot diameters and gap sizes are evaluated. The starting point for the spot diameter can be estimated from the limit of detection (LOD) of cholesterol reported in a previous publication ${ }^{21}$. The LOD for cholesterol was varied over three orders of magnitude. If the signal is proportional to the desorption area, that is quadratic to the radius, roughly 
$30 \mu \mathrm{m}$ in diameter seem to work as copper spots. As the glass isolates the heat, gaps between $5 \mu \mathrm{m}$ and $20 \mu \mathrm{m}$ were tested. As seen in Supplementary Fig. S10-S12, a gap of $10 \mu \mathrm{m}$ with spot diameters of $30 \mu \mathrm{m}$ is optimal. The grid is shown in Supplementary, Fig. S8. These results will be confirmed visually using mouse liver tissue on the grid of copper spots.

Fig. 2 shows a microscopic image of a part of the microtome cut, close to an edge with diode laser application in spot-by-spot mode. Plain copper spots, laser-applied copper spots and the animal tissue in the lower part of the image are highlighted. This observation fits to the previously taken considerations including the confinement of the heat propagation and spot diameters.

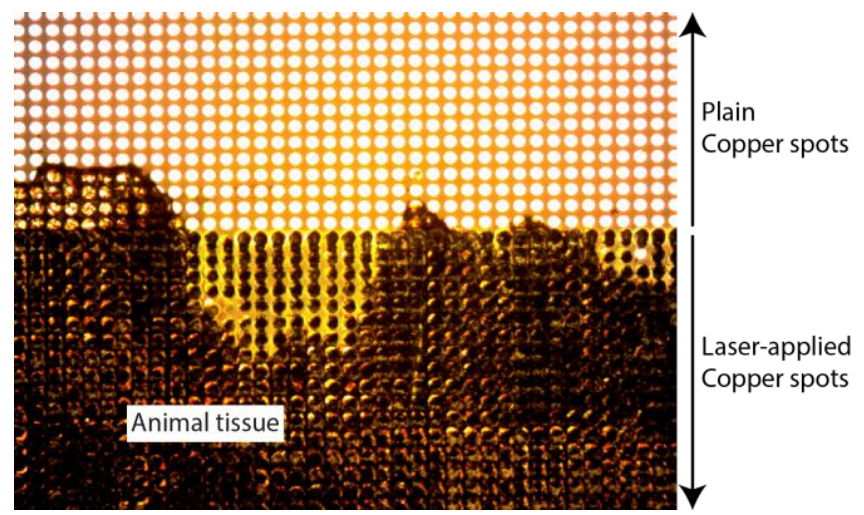

Fig. 2. Microscopic image of the screened mouse liver microtome cut. The figure shows distinct regions of the animal tissue, plain and laser-applied copper spots.

The XIC of dehydrated cholesterol ( $\delta m / z, 368.6-370.1)$ was taken for each column in Fig. 3. A typical chromatogram of spot-by-spot scanning is shown in Fig. 3a. Each signal is separated by a gap that origins from moving the stage to the next spot. In this period, the laser shutter is closed. In general, spots without the tissue only show a neglectable ion response. Proper signal slowly emerges on the borders of the tissue. The lower signal is mostly related to two aspects. On the one hand, the tissue partially overlaps with the copper spots rather than fully cover them. On the other hand, the tissue thickness assumingly decreases on the sides. Besides, the gap between copper spots, the signal of a single spot and information related to the data analysis are highlighted. This information is used to separate a valid signal from the plasma ion background. The basic functionality is described in a flow chart in Supplementary, Fig. S1 and $\mathrm{S} 2$ and in detail in the experimental methods. In total, a region of interest (ROI) of $5 \mathrm{~mm} \times 5 \mathrm{~mm}$ on that copper grid translates into 125 individually recorded files. A full measurement takes roughly 7 hours with a single laser application time of one second to each spot with roughly 16000 copper spots. Without the proposed software, several seconds are generally required for the initialization of the data files plus another few seconds per measurement with otherwise equal conditions. Therefore, more than two days of experimental duration would be required for a single image if the algorithm was not used. It is worth noting that numerous imaging techniques offer experimental times of less than an hour. However, this is not a method-specific effect, but mostly related to the sampling rate of the mass analyzer.
In Fig. 3b, the substrate is screened using line scanning. In this mode, the stage moves at a constant speed, whose parameters are matched to the spot-by-spot screening. Notably, the copperfree glass area also yield a signal response, which is denoted as "matrix background". This signal is linked to absorption of the laser light on the microtome cut. In contrast to Fig. 3a, the ion response is lower. This detail will be discussed in the following.

In general, scanning speeds above $150 \mu \mathrm{m} / \mathrm{s}$ are possible to achieve an ion response above noise level. However, higher scan speeds reduce the amount of spectra recorded. For instance, with a fixed sampling rate of $60 \mathrm{~Hz}$ and a scanning speed of $150 \mu \mathrm{m} / \mathrm{s}, 12$ mass spectra are recorded for a single spot. With speeds above $450 \mu \mathrm{m} / \mathrm{s}$, less than 4 spectra would be recorded for each spot, losing the statistic character of the experiment. In addition, the signals coincide at those speeds and complicate the spot allocation. Likewise, a scan speed below $30 \mu \mathrm{m} / \mathrm{s}$ increases the number of spectra recorded, but also leads to an increased experimental time without the benefits of a better spatial resolution. Hence, an optimal scanning speed is a trade-off of measurement time versus sampling rate, spatial resolution and signal intensity. Another interesting observation is that the ion signal in spot-by-spot scanning is often splitted into two separate signal peaks. However, this does not apply to line scanning,. It is assumed that the increase in the ion signal within a second of application time emerges from different temperatures inside the $30 \mu \mathrm{m}$ spot.

This obviously requires for an automated post-processing data script, as described in the experimental methods. The final image for the target analysis of cholesterol $(\mathrm{m} / \mathrm{z}$ 369.7) in a mouse liver is shown in Fig. 4. 
a)

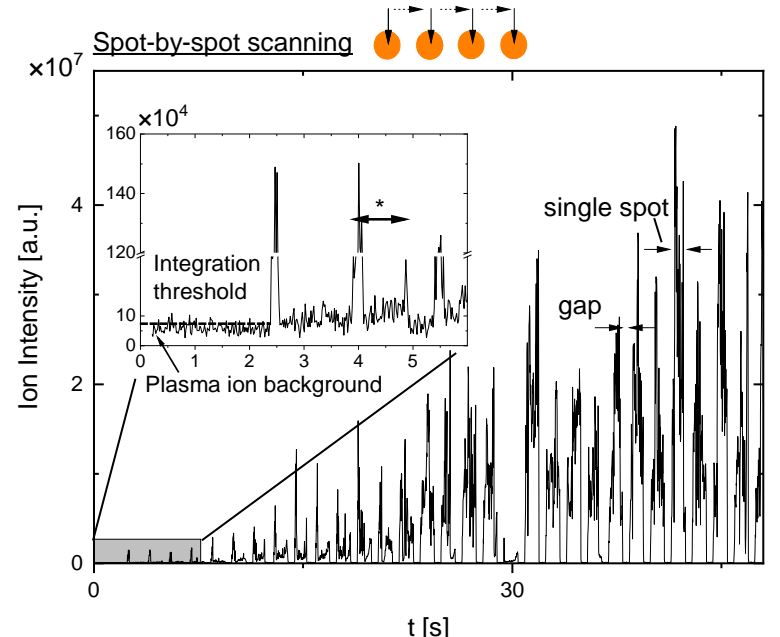

b)

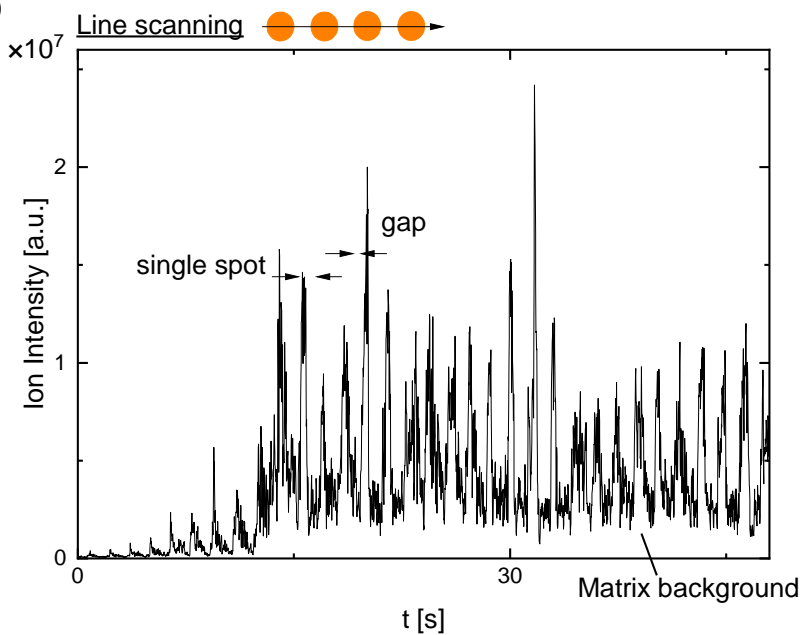

Fig. 3. Extracted ion current ( $\delta \mathrm{m} / z, 368.6-370.1$ ) of dehydrated cholesterol using spot-by-spot (a) and line scanning (b). A possible intensity threshold used for integration of the peaks as well as the gap originating from stage movement is shown. The integration threshold is usually lower. The indication is chosen to allow the reader to get the idea of the post processing algorithm.

Fig. 4 maps the unfolded ion intensities of each spot to the absolute positions of the spot on the grid. In general, a rather homogenous image can be confirmed, with aggregations of cholesterol on the top side of the tissue. The homogeneity of the tissue seems plausible, as cholesterol is needed for synthesis in each cell. The homogeneity of this tissue is proven by hematoxylin and eosin stains (H\&E stains), which are produced by the recipe of Supplementary, Table S7 and shown in Supplementary Fig. S13-S14. The signal deviation is thus a product of the plasma noise.

The borders of the tissue can be clearly identified. The reconstructed image matches to the morphology of the tissue, which can be seen in Supplementary Fig. S15. The highlighted part of the reconstructed image was also shown in Fig. 2.

The edges of the tissue may show fringes. This can be seen in the lower right part of the reconstructed image. This is not an effect by the post processing algorithm, but from the tissue itself, also observed with some H\&E stains, such as seen in Supplementary Fig. S16.
Furthermore, another conclusion that can be drawn is that no sample deposition take place after laser desorption, as there is a clear cut of ion signal and plasma noise on the border of the tissue. In this sense, this image proves the suitability of the algorithm for data analysis.

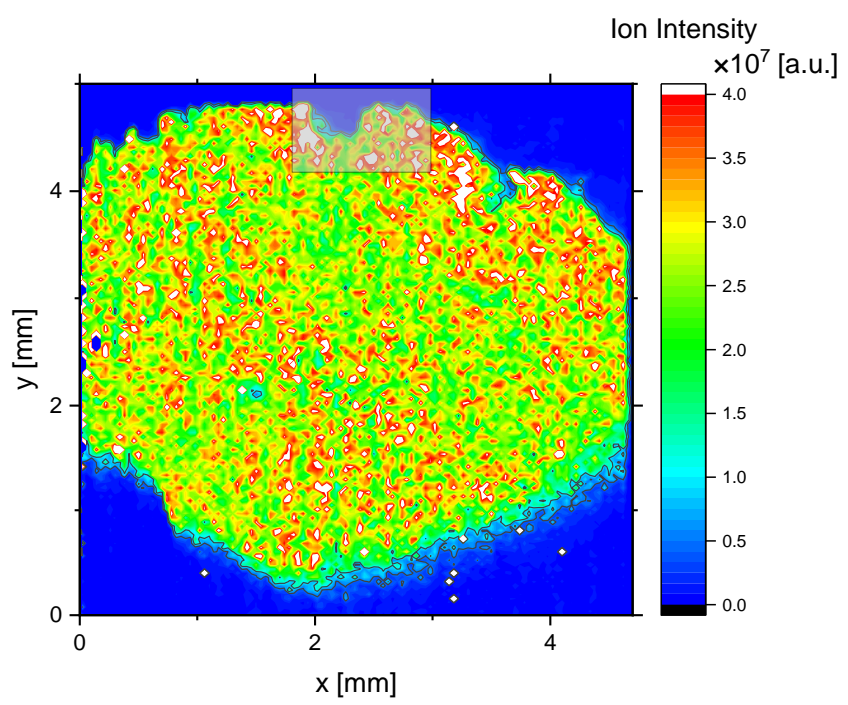

Fig. 4. Image of dehydrated cholesterol $(\mathrm{m} / \mathrm{z}, 369.7)$ a mouse liver microtome cut using the laser desorption flexible microtube plasma ionization interface. A striking region of the cut can be safely assigned to a microscopic image shown in Fig. 3.

In a previous publication ${ }^{22}$, a bovine liver extract was used as a complex standard with a similar laser desorption/plasma ionization setup. These results have shown the possibility for lipid profiling using a laser-plasma hyphenated mass spectrometric method.

In Fig. 5, this method was applied to microtome cuts of mouse liver, kidney and brain using a Paul ion trap (LCQ Deca XP, Thermo Fisher, USA) on pure copper-glass substrates with the same experimental conditions of the imaging. In all three cuts, free cholesterol $(\mathrm{m} / \mathrm{z}, 369.8)$ can be identified as the base peak. Further, all three tissues show phospholipids and glycerophospholipids around $m / z 600$.

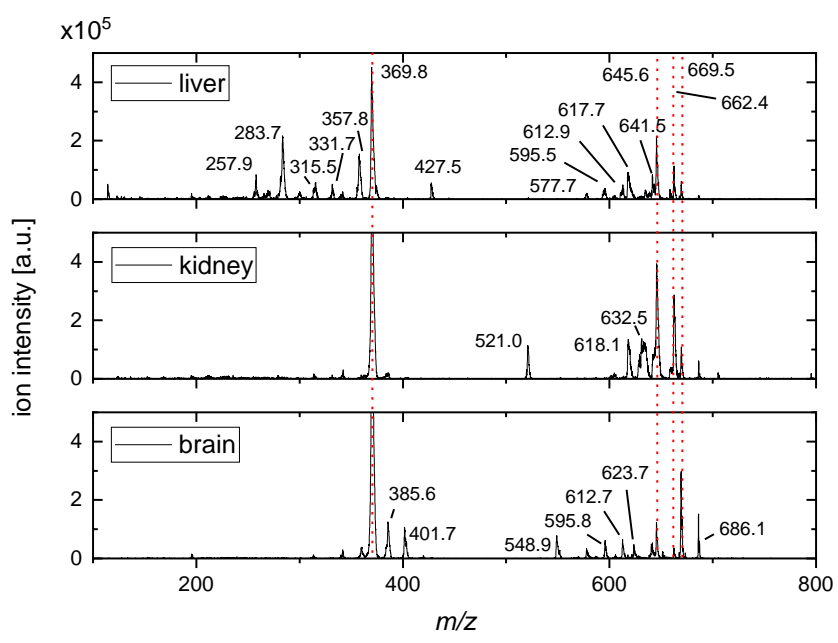

Fig. 5. Mass spectra of microtome cuts from mouse liver, kidney and brain using laser desorption, plasma ionization. 
Some of these lipids, $\mathrm{m} / \mathrm{z}, 645.6,662.4$ and 669.5 , can be identified in all three tissues. However, many other compounds are tissue-specific, such as $m / z, 521.0$ in the kidney, $m / z, 385.6$ in the brain or $m / z, 283.7$ in the liver. In this sense, numerous compounds remain promising for imaging purposes. However, a larger imaging study of these compounds will be postponed to future work.

In concern of imaging applications, the spatial resolution is a critical parameter for imaging. For instance, the best spatial resolution in MALDI was achieved by adding a second laser for post ionization in a method named t-MALDI- $2^{37,38}$, resolving at as low as $600 \mathrm{~nm}$. Ambient mass spectrometry methods seem to achieve micrometer resolution at maximum. In addition, the thermal confinement on the desorption substrate seems to be a key factor, assumingly correlated to continuous-wave versus pulsed laser operation. Better spatial resolution for this method requires small laser light divergence and shorter laser pulses. However, the lasers output and the divergence of laser diodes are linked to the geometry of the emitter. The laser output power generally increases with increasing emitter sizes. In fact, emitters are stacked for that particular reason. On the other hand, an increasing emitter causes formation of more lateral modes or waveguides inside the cavity, which impact the quality of the laser spot. Finally, the shorter pulses are inherently linked to lower laser output powers per pulse. Other ambient imaging methods in mass spectrometry are listed in Table 1.

AP-MALDI is the direct development of conventional MALDI to ambient conditions. Despite the operating conditions, the sample preparation is still a complex process. Electrospray- and plasma-based methods have also proven to be suitable for desorption. The spatial resolution in DESI has increased over the past years up to several tenths of micrometers using concentric solvent sprays ${ }^{39}$, decreased tips of the capillary ${ }^{40}$ or air flowassisted ionization DESI (AFA-DESI) ${ }^{41}$ in line scanning.

On the plasma-side, LTPs have been successfully used for imaging concerns ${ }^{13,14}$, also coupled with $\mathrm{TLC}^{10}$. Commonly, pulsed lasers are used for imaging. However, the recent publication by the Winkler group ${ }^{13}$ reports on a cw blue laser diode. The authors provide a pixel size of $100 \times 100 \mu \mathrm{m}^{2}$. In this sense, pulsed lasers may lead to a better thermal confinement in direct comparison to their cw counterparts, but also push the desorption towards ablation. This effect could be confirmed in Supplementary, Fig. S9 as well.

The use of cw lasers is not solely limited to LTPs. For instance, FAPA $^{9}$ has been also coupled to blue laser diodes operated in $\mathrm{cW}$ and combined with TLC. In other plasma-based methods such as PALDI ${ }^{12}$ or DART ${ }^{7}$, pulsed lasers were preferably used.

In similar methods such as the previously mentioned LDTDAPCI-MSMS, a corona discharge is used in insource operation with a heated gas flow for transport of the analyte molecules, mostly coupled to infrared lasers. These lasers are applied to well plates for the desorption of extracted samples. Despite the imaging application of this work, the substrate is placed closed to the inlet of the mass spectrometer, with the plasma fixed at a few hundred micrometer in front of it. In this sense, no guided transport is required. From the physical side, the laser is solely used for the desorption and a flexible micro plasma for ionization.

Other methods that use metals to assist desorption are based on silver ${ }^{18}$ or gold ${ }^{19}$ (layers $^{20}$ ). In the metal-assisted LDI methods, the metal is directly sputtered on the sample. For that reason, the laser firstly ablates the metal layer and then the target analyte ions. The significant difference of our method to the shown work is that the sputtered metal ions reach the substrate with a defined kinetic energy at a fixed surface temperature. In contrast, the substrate are fully prepared for the experiment before sample preparation. As a result, the referenced publications detect metal clusters and adducts. However, the concept is inherently different from MALDI.

Some of the aforementioned methods (and sometimes even seen in MALDI methods) commonly estimate the spatial resolution by correlating the scan speed to the sampling rate of the mass spectrometer. It is worth noting that this estimation may show significant bias to lower resolution. For instance, correlating the sampling rate of this mass spectrometer to the used scan speed, $30 \mu \mathrm{m} / \mathrm{s} / 60 \mathrm{~Hz}=500 \mathrm{~nm}$ were assumingly resolved.

Table 1. Comparison of ambient imaging methods in mass spectrometry.

\begin{tabular}{|l|l|l|l|l|l|}
\hline Method & $\begin{array}{l}\text { Desorption/Abla- } \\
\text { tion }\end{array}$ & Ionization & Matrix & $\begin{array}{l}\text { Resolution } \\
{[\mu \mathrm{m} \times \mu \mathrm{m}]}\end{array}$ & Reference \\
\hline AP-MALDI & Pulsed Laser & MALDI & Yes & $\begin{array}{l}1.4 \times 1.4 \\
20 \times 20\end{array}$ & $\begin{array}{l}3, \\
42\end{array}$ \\
\hline DESI & Spray & ESI & No & $\begin{array}{l}<500 \times 500 \\
40 \times 500\end{array}$ & $\begin{array}{l}43, \\
39\end{array}$ \\
\hline LTP & Plasma & Plasma & No & $250 \times 250$ & 44 \\
\hline LD-LTP & $\begin{array}{l}\text { cw Laser, partially } \\
\text { TLC }\end{array}$ & Plasma & Both & $25 \times 25$ & 13,14 \\
\hline $\begin{array}{l}\text { LDTD-APCI- } \\
\text { MSMS }\end{array}$ & (Probably) cw Laser & APCI & No & Not given & $15-17$ \\
\hline Metal-assisted LDI & Pulsed laser & $\begin{array}{l}\text { Partially } \\
\text { MALDI }\end{array}$ & Both & $\begin{array}{l}\text { Ca. } \\
100 \mu \mathrm{m} \\
\text { lateral }\end{array}$ & 18,19 \\
\hline
\end{tabular}




\begin{tabular}{|l|l|l|l|l|l|}
\hline & & & & $\begin{array}{l}\text { without } \\
\text { over- } \\
\text { sampling }\end{array}$ & \\
\hline LA-DART & Pulsed Laser & DART & No & $\begin{array}{l}4-250 \mu \mathrm{m} \\
\text { lateral }\end{array}$ & 7,8 \\
\hline LA-FAPA & $\begin{array}{l}\text { Pulsed and cw La- } \\
\text { ser, partially TLC }\end{array}$ & FAPA & No & $\begin{array}{l}\text { c.a. } 20 \mu \mathrm{m} \\
\text { lateral }\end{array}$ & 6,9 \\
\hline TLC-PALDI & Pulsed Laser, TLC & PALDI & No & $60 \times 60$ & 11,12 \\
\hline DLD-PI MSI & Pulsed Laser & Plasma & No & $40 \times 40$ & This work \\
\hline
\end{tabular}

\section{Concluding remarks}

The work highlights a systematic development of imaging using a pulsed diode laser for desorption and the flexible microtube plasma for ionization. Effects from crucial parameters such as the laser pulse frequency or the laser pulse width are studied step-by-step to get a control on the thermal confinement on the substrate. In addition, a novel design of the desorption substrate with a grid of copper spots and calibration markers was presented, that was optimized to the imaging application. On that basis, the laser is applied to each copper spot on the grid controlled by a self-made, LabVIEW-based software in all three dimensions. Each line of spots is saved into a single file, which compresses the total data size and the experimental duration. For that reason, an algorithm written in Python is developed, that automatically unfolds and reconstructs the data, even for low abundant signals. As a result, the image can be screened at lower sampling rates and thus higher mass resolution.

An interesting property of imaging is the screening method itself. Line scanning at a constant speed is often preferred over spot-by-spot scanning, as it significantly reduces the experimental duration. However, the differences are only rarely discussed if they are mentioned at all. In this work, these two modes are compared with one another, with one difference being the signal-to-noise ratio.

A spot diameter of $30 \mu \mathrm{m}$ can be resolved, being separated by a $10 \mu \mathrm{m}$ gap. The imaging capability is proven by screening a mouse liver sample for dehydrated cholesterol directly from the tissue, an analyte well-known from previous work. For validation, the result is compared to the morphology of the tissue, proving good agreement with one another. Further characteristics of the tissue are evaluated from H\&E stains. Finally, a view on future analysis of further molecules from other tissue is given.

In contrast to some of the aforementioned methods, no conventional matrices or sample extraction, thin-layer chromatography, vacuum conditions or an ablation cell are required. In fact, the material is not ablated from the substrate at all, but trapped inside. This effect origins from the sandwich design of the substrate. Other benefits are the ambient air operation and a comparably short analysis time in a modular setup design.

\section{Associated Content Author Contributions}

All authors have given approval to the final version of the manuscript.

Notes

The authors declare no competing financial interest.

\section{CRediT Authorship Contribution Statement}

A. Knodel: Conceptualization, Methodology, Validation, Investigation, Resources, Writing - Original Draft, Review \& Editing, Visualization, Software U. Marggraf: Resources (Substrates) E. Hoffmann-Posorske: Resources S. Burhenn: Software, Writing Review \& Editing S. Brandt: Conceptualization, Methodology (F $\mu$ TP), Software, Writing - Review \& Editing N. Ahlmann: Resources (Laser safety \& operation) D. Foest: Resources K. Lorenz: Project administration, Funding acquisition J. Franzke: Writing Review \& Editing, Supervision, Project administration, Funding acquisition

\section{Acknowledgement}

The financial support from the Ministerium für Kultur und Wissenschaft des Landes Nordrhein-Westfalen, Der Regierende Bürgermeister von Berlin, Senatskanzlei Wissenschaft und Forschung, the Bundesministerium für Bildung und Forschung, and the Deutsche Forschungsgemeinschaft is acknowledged gratefully. The authors want to thanks the Miniaturization group, Dr. Bienvenida GilbertLópez (Department of Physical and Analytical Chemistry, Universidad de Jaén), Prof. Dr.-Ing. Stefan Tappertzhofen (Chair for Micro- und Nanoelectronics, TU Dortmund) and Irina Ribeiro Bas and Stefanie Dörr for their support and lively discussions.

\section{Supporting Information.}

The Supporting Information is available free of charge on the ACS Publications website. It includes flow charts for the software, data on the laser spot, laser pulses with different laser parameters, a recipe of H\&E stains, an image of the used copper grid, a grid of copper spots with different diameters and gaps, images of the $\mathrm{H} \& \mathrm{E}$ stains and a microscopic image of the spot-by-spot screened liver tissue.

\section{References}

(1) Castaing, R.; Slodzian, G. Microanalysis by secondary ionic emission. J. Microscopy, 1962, 395-410.

(2) Karas, M.; Bachmann, D.; Hillenkamp, F. Influence of the wavelength in high-irradiance ultraviolet laser desorption 
mass spectrometry of organic molecules. Anal. Chem. 1985, 57 (14), 2935-2939. DOI: 10.1021/ac00291a042.

(3) Kompauer, M.; Heiles, S.; Spengler, B. Atmospheric pressure MALDI mass spectrometry imaging of tissues and cells at $1.4\{\backslash$ textmu $\} \mathrm{m}$ lateral resolution. Nat. Methods 2017, 14 (1), 90-96. DOI: 10.1038/nmeth.4071.

(4) Garikapati, V.; Karnati, S.; Bhandari, D. R.; BaumgartVogt, E.; Spengler, B. High-resolution atmospheric-pressure MALDI mass spectrometry imaging workflow for lipidomic analysis of late fetal mouse lungs. Scientific reports 2019, 9 (1), 3192. DOI: 10.1038/s41598-019-39452-3.

(5) Jackson, S. N.; Muller, L.; Roux, A.; Oktem, B.; Moskovets, E.; Doroshenko, V. M.; Woods, A. S. AP-MALDI Mass Spectrometry Imaging of Gangliosides Using 2,6-Dihydroxyacetophenone. Journal of the American Society for Mass Spectrometry 2018, 29 (7), 1463-1472. DOI: 10.1007/s13361018-1928-8.

(6) Shelley, J. T.; Ray, S. J.; Hieftje, G. M. Laser ablation coupled to a flowing atmospheric pressure afterglow for ambient mass spectral imaging. Anal. Chem. 2008, 80 (21), 83088313. DOI: 10.1021/ac801594u. Published Online: Oct. 1, 2008

(7) Fowble, K. L.; Teramoto, K.; Cody, R. B.; Edwards, D.; Guarrera, D.; Musah, R. A. Development of "Laser Ablation Direct Analysis in Real Time Imaging" Mass Spectrometry: Application to Spatial Distribution Mapping of Metabolites Along the Biosynthetic Cascade Leading to Synthesis of Atropine and Scopolamine in Plant Tissue. Anal. Chem. 2017, 89 (6), 3421-3429. DOI: 10.1021/acs.analchem.6b04137. Published Online: Mar. 9, 2017.

(8) Galhena, A. S.; Harris, G. A.; Nyadong, L.; Murray, K. K.; Fernández, F. M. Small molecule ambient mass spectrometry imaging by infrared laser ablation metastable-induced chemical ionization. Anal. Chem. 2010, 82 (6), 2178-2181. DOI: $10.1021 /$ ac902905v.

(9) Cegłowski, M.; Smoluch, M.; Reszke, E.; Silberring, J.; Schroeder, G. Flowing atmospheric pressure afterglow combined with laser ablation for direct analysis of compounds separated by thin-layer chromatography. Anal. Bioanal. Chem. 2016, 408 (3), 815-823. DOI: 10.1007/s00216-015-9165-5. Published Online: Nov. 12, 2015.

(10) García-Rojas, N. S.; Moreno-Pedraza, A.; Rosas-Román, I.; Ramírez-Chávez, E.; Molina-Torres, J.; Winkler, R. Mass spectrometry imaging of thin-layer chromatography plates using laser desorption/low-temperature plasma ionisation. Analyst 2020, 145 (11), 3885-3891. DOI: 10.1039/D0AN00446D. Published Online: Apr. 16, 2020.

(11) Zhang, J.; Zhou, Z.; Yang, J.; Zhang, W.; Bai, Y.; Liu, H. Thin layer chromatography/plasma assisted multiwavelength laser desorption ionization mass spectrometry for facile separation and selective identification of low molecular weight compounds. Anal. Chem. 2012, 84 (3), 1496-1503. DOI: 10.1021/ac202732y. Published Online: Jan. 30, 2012.

(12) Feng, B.; Zhang, J.; Chang, C.; Li, L.; Li, M.; Xiong, X.; Guo, C.; Tang, F.; Bai, Y.; Liu, H. Ambient mass spectrometry imaging: plasma assisted laser desorption ionization mass spectrometry imaging and its applications. Anal. Chem. 2014, 86 (9), 4164-4169. DOI: 10.1021/ac403310k.

(13) Moreno-Pedraza, A.; Rosas-Román, I.; Garcia-Rojas, N. S.; Guillén-Alonso, H.; Ovando-Vázquez, C.; Díaz-Ramírez, D.; Cuevas-Contreras, J.; Vergara, F.; Marsch-Martínez, N.; Molina-Torres, J.; Winkler, R. Elucidating the Distribution of Plant Metabolites from Native Tissues with Laser Desorption
Low-Temperature Plasma Mass Spectrometry Imaging. Analytical chemistry 2019, 91 (4), 2734-2743. DOI:

10.1021/acs.analchem.8b04406.

(14) Yan, B.; Murta, T.; Elia, E. A.; Steven, R. T.; Bunch, J. Direct Tissue Mass Spectrometry Imaging by Atmospheric

Pressure UV-Laser Desorption Plasma Postionization. Journal of the American Society for Mass Spectrometry 2020. DOI: 10.1021/jasms.0c00315.

(15) Swales, J. G.; Gallagher, R.; Peter, R. M. Determination of metformin in mouse, rat, dog and human plasma samples by laser diode thermal desorption/atmospheric pressure chemical ionization tandem mass spectrometry. J. Pharm. Biomed. Anal. 2010, 53 (3), 740-744. DOI:

10.1016/j.jpba.2010.04.033. Published Online: May. 2, 2010.

(16) Lanshoeft, C.; Heudi, O.; Leuthold, L. A.; Schlotterbeck, G.; Elbast, W.; Picard, F.; Kretz, O. Laser diode thermal desorption atmospheric pressure chemical ionization tandem mass spectrometry applied for the ultra-fast quantitative analysis of BKM120 in human plasma. Anal. Bioanal. Chem. 2014, 406 (22), 5413-5423. DOI: 10.1007/s00216-014-7966-6. Published Online: Jun. 24, 2014.

(17) Borik, A.; Staňová, A. V.; Brooks, B. W.; Grabicová, K.; Randák, T.; Grabic, R. Determination of citalopram in fish brain tissue: benefits of coupling laser diode thermal desorption with low- and high-resolution mass spectrometry. Anal. Bioanal. Chem. 2020, 412 (18), 4353-4361. DOI: 10.1007/s00216-020-02672-y. Published Online: May. 6, 2020 .

(18) Dufresne, M.; Thomas, A.; Breault-Turcot, J.; Masson, J.-F.; Chaurand, P. Silver-assisted laser desorption ionization for high spatial resolution imaging mass spectrometry of olefins from thin tissue sections. Anal. Chem. 2013, 85 (6), 33183324. DOI: 10.1021/ac3037415. Published Online: Mar. 7, 2013.

(19) Chen, L. C.; Mori, K.; Hori, H.; Hiraoka, K. Au-assisted visible laser MALDI. International Journal of Mass Spectrometry 2009, 279 (1), 41-46. DOI:

10.1016/j.ijms.2008.10.005.

(20) Lin, J.-Y.; Chen, T.-Y.; Chen, J.-Y.; Chen, Y.-C. Multilayer gold nanoparticle-assisted thermal desorption ambient mass spectrometry for the analysis of small organics. Analyst 2010, 135 (10), 2668-2675. DOI: 10.1039/C0AN00157K. Published Online: Aug. 19, 2010.

(21) Knodel, A.; Marggraf, U.; Ahlmann, N.; Brandt, S.; Foest, D.; Gilbert-López, B.; Franzke, J. Standardization of Sandwich-Structured Cu-Glass Substrates Embedded in a Flexible Diode Laser-Plasma Interface for the Detection of Cholesterol. Anal. Chem. 2020, 92 (6), 4663-4671. DOI: 10.1021/acs.analchem.0c00311.

(22) Knodel, A.; Foest, D.; Brandt, S.; Ahlmann, N.; Marggraf, U.; Gilbert-López, B.; Franzke, J. Detection and Evaluation of Lipid Classes and Other Hydrophobic Compounds Using a Laser Desorption/Plasma Ionization Interface. Anal. Chem. 2020. DOI: 10.1021/acs.analchem.0c03839.

(23) Zavalin, A.; Yang, J.; Hayden, K.; Vestal, M.; Caprioli, R. M. Tissue protein imaging at $1-\{\backslash$ textmu $\} \mathrm{m}$ laser spot diameter for high spatial resolution and high imaging speed using transmission geometry MALDI TOF MS. Analytical and bioanalytical chemistry 2015, 407 (8), 2337-2342. DOI: 10.1007/s00216-015-8532-6.

(24) Berry, K. A. Z.; Hankin, J. A.; Barkley, R. M.; Spraggins, J. M.; Caprioli, R. M.; Murphy, R. C. MALDI imaging 
of lipid biochemistry in tissues by mass spectrometry. Chemical reviews 2011, 111 (10), 6491-6512. DOI:

$10.1021 / \mathrm{cr} 200280 \mathrm{p}$.

(25) Sullards, M. C.; Liu, Y.; Chen, Y.; Merrill, A. H. Analysis of mammalian sphingolipids by liquid chromatography tandem mass spectrometry (LC-MS/MS) and tissue imaging mass spectrometry (TIMS). Biochimica et biophysica acta 2011, 1811 (11), 838-853. DOI: 10.1016/j.bbalip.2011.06.027. (26) Sugiura, Y.; Setou, M. Selective imaging of positively charged polar and nonpolar lipids by optimizing matrix solution composition. Rapid communications in Mass Spectrometry 2009, 23 (20), 3269-3278. DOI: 10.1002/rcm.4242.

(27) Vance, J. E. Dysregulation of cholesterol balance in the brain: contribution to neurodegenerative diseases. Disease models \& mechanisms 2012, 5 (6), 746-755. DOI:

10.1242/dmm.010124. Published Online: Oct. 12, 2012.

(28) Fessler, M. B. Regulation of Adaptive Immunity in Health and Disease by Cholesterol Metabolism. Curr. Allergy Asthma Rep. (Current allergy and asthma reports) 2015, 15 (8), 48. DOI: 10.1007/s11882-015-0548-7.

(29) Arguello, G.; Balboa, E.; Arrese, M.; Zanlungo, S. Recent insights on the role of cholesterol in non-alcoholic fatty liver disease. Biochimica et biophysica acta 2015, 1852 (9), 1765-1778. DOI: 10.1016/j.bbadis.2015.05.015.

(30) Cologna, S. M. Mass Spectrometry Imaging of Cholesterol. Adv. Exp. Med. Biol. (Advances in experimental medicine and biology) 2019, 1115, 155-166. DOI: 10.1007/978-3030-04278-3_7.

(31) Allain, F. PyMSFileReader. https://github.com/frallain/\pymsfilereader (accessed 2020-12-01).

(32) Thermo Fisher Scientific Inc. MSFileReader. http:// tools.thermofisher.com/content/sfs/manuals/Man-XCALI97542-MSFileReader-30-Ref-ManXCALI97542-A-EN.pdf (accessed 2020-12-01).

(33) Zaber Technologies Inc. Manuals T-LSM. https:// www.zaber.com/wiki/Manuals/T-LSM (accessed 2020-10-28). (34) Lasertack GmbH. LDM-450-1600-C Blue Laser Module. https://lasertack.com/Media/Uploaded/Datasheets_software/ LDM-450-1600-C.pdf (accessed 2020-06-18).

(35) Brandt, S.; Klute, F. D.; Schütz, A.; Marggraf, U.; Drees, C.; Vogel, P.; Vautz, W.; Franzke, J. Flexible Microtube Plasma (F-\{ \textmu $\}$ TP) as an Embedded Ionization Source for a Microchip Mass Spectrometer Interface. Anal. Chem. 2018, 90 (17), 10111-10116. DOI: 10.1021/acs.analchem.8b01493.

(36) Thorlabs Inc. NPL Series of Nanosecond Pulsed Lasers. www.thorlabs.com $\_s d . c f m ? f i l e N a m e=T T N 122959$ -

D02.pdf\&partNumber=NPL45C (accessed 2020-12-09).

(37) Niehaus, M.; Soltwisch, J.; Belov, M. E.; Dreisewerd, K. Transmission-mode MALDI-2 mass spectrometry imaging of cells and tissues at subcellular resolution. Nat. Methods $\mathbf{2 0 1 9}$, 16 (9), 925-931. DOI: 10.1038/s41592-019-0536-2.

(38) Woods, A. S.; Jackson, S. N.; Lewis, E. K.; Egan, T.; Muller, L.; Tabet, J.-C.; Schultz, J. A. MALDI/post ionization-ion mobility mass spectrometry of noncovalent complexes of dopamine receptors' epitopes. J. Proteome Res. 2013, 12 (4), 1668-1677. DOI: 10.1021/pr301004w.

(39) Tillner, J.; Wu, V.; Jones, E. A.; Pringle, S. D.; Karancsi, T.; Dannhorn, A.; Veselkov, K.; McKenzie, J. S.; Takats, Z. Faster, More Reproducible DESI-MS for Biological Tissue Imaging. J. Am. Soc. Mass Spectrom. Chem. 2017, 28 (10), 2090-2098. DOI: 10.1021/jasms.8b05382.

(40) Laskin, J.; Heath, B. S.; Roach, P. J.; Cazares, L.; Semmes, O. J. Tissue imaging using nanospray desorption electrospray ionization mass spectrometry. Anal. Chem. 2012, 84 (1), 141-148. DOI: 10.1021/ac2021322. Published Online: Dec. 1, 2011.

(41) Luo, Z.; He, J.; Chen, Y.; He, J.; Gong, T.; Tang, F.; Wang, X.; Zhang, R.; Huang, L.; Zhang, L.; Lv, H.; Ma, S.; Fu, Z.; Chen, X.; Yu, S.; Abliz, Z. Air flow-assisted ionization imaging mass spectrometry method for easy whole-body molecular imaging under ambient conditions. Anal. Chem. 2013, 85 (5), 2977-2982. DOI: 10.1021/ac400009s. Published Online: Feb. 20, 2013.

(42) Elia, E. A.; Niehaus, M.; Steven, R. T.; Wolf, J.-C.; Bunch, J. Atmospheric Pressure MALDI Mass Spectrometry Imaging Using In-Line Plasma Induced Postionization. Anal. Chem. 2020, 92 (23), 15285-15290. DOI: 10.1021/acs.analchem.0c03524.

(43) Wiseman, J. M.; Ifa, D. R.; Song, Q.; Cooks, R. G. Tissue imaging at atmospheric pressure using desorption electrospray ionization (DESI) mass spectrometry. Angew. Chem. Int. Ed. 2006, 45 (43), 7188-7192. DOI:

10.1002/anie.200602449.

(44) Liu, Y.; Ma, X.; Lin, Z.; He, M.; Han, G.; Yang, C.; Xing, Z.; Zhang, S.; Zhang, X. Imaging mass spectrometry with a low-temperature plasma probe for the analysis of works of art. Angew. Chem. Int. Ed. 2010, 49 (26), 4435-4437. DOI: 10.1002/anie.200906975. 
Graphic entry for TOC

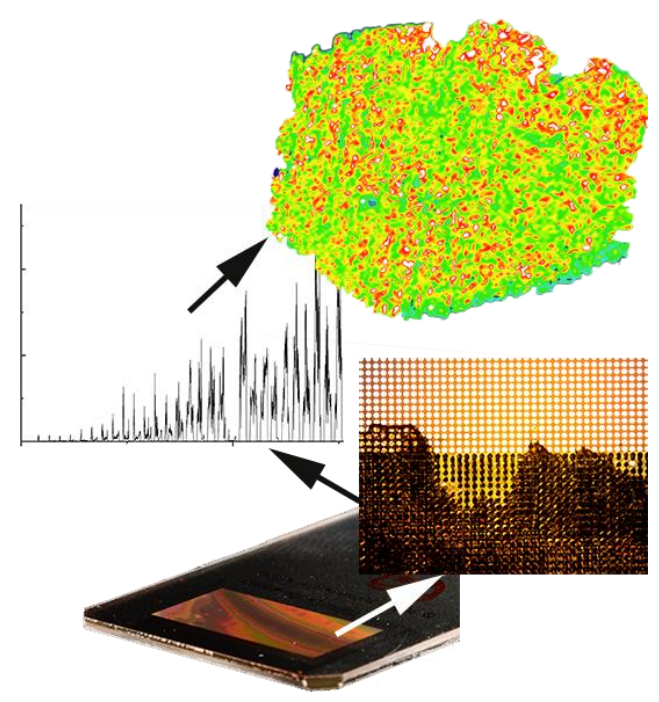

